\title{
BMJ Open Colorectal cancer Outcomes in people with Severe Mental Illness Cohort (COSMIC): a protocol for an Australian retrospective cohort using linked administrative data
}

\author{
Melinda M Protani (D) , ', Susan J Jordan, ${ }^{1,2}$ Bradley J Kendall, ${ }^{3,4}$ Dan Siskind,,${ }^{3,5}$ \\ David Lawrence (D) ," Grant Sara (D) , ", Lisa Brophy, ${ }^{9,10}$ Steve Kisely (D) ${ }^{3,5}$
}

To cite: Protani MM, Jordan SJ, Kendall BJ, et al. Colorectal cancer Outcomes in people with Severe Mental IIIness Cohort (COSMIC): a protocol for an Australian retrospective cohort using linked administrative data. BMJ Open 2021;11:e044737. doi:10.1136/ bmjopen-2020-044737

- Prepublication history for this paper is available online. To view these files, please visit the journal online (http://dx.doi. org/10.1136/bmjopen-2020044737).

Received 11 September 2020 Accepted 21 May 2021

Check for updates

(C) Author(s) (or their employer(s)) 2021. Re-use permitted under CC BY-NC. No commercial re-use. See rights and permissions. Published by BMJ.

For numbered affiliations see end of article.

Correspondence to

Dr Steve Kisely;

s.kisely@uq.edu.au

\section{ABSTRACT}

Introduction Colorectal cancer (CRC) mortality is significantly higher in those with severe mental illness (SMI) compared with the general population, despite similar incidence rates, suggesting that barriers to optimal screening and cancer care may contribute to disparities in CRC mortality in those with SMI. This study aims to compare participation in Australia's National Bowel Cancer Screening Programme (NBCSP) in those with SMl and those in the general population. We will also investigate treatment pathways after diagnosis to determine whether treatment variations could explain differences in CRC mortality.

Methods and analysis We will undertake a retrospective cohort study of Australians using linked administrative data to assess differences in screening and cancer care between those with and without SMI, aged 50-74 years on or after 1 January 2006. People with SMI will be defined using antipsychotic medication prescription data. The comparison group will be people enrolled in Medicare (Australia's universal healthcare system) who have not been prescribed antipsychotic medication. Data on outcomes (NBCSP participation, follow-up colonoscopy, CRC incidence and CRC-cause and all-cause mortality) and confounders will be obtained from national-based and state-based administrative health datasets. All people in New South Wales, aged 50-74 with a new diagnosis of CRC on or after 1 January 2006, will be ascertained to examine stage at diagnosis and cancer treatment in those with and without SMI. Poisson regression will be used to calculate incidence rates and rate ratios for each outcome. Ethics and dissemination Ethics approval has been obtained from the University of Queensland Human Research Ethics Committee, the Australian Institute of Health and Welfare Ethics Committee and data custodians from every Australian State/Territory. Findings will be disseminated via publications in peer-reviewed journals and presented at appropriate conferences.

Trial registration number ACTRN12620000781943.

\section{INTRODUCTION}

Mortality rates in patients with severe mental illnesses (SMI), such as schizophrenia and

\section{Strengths and limitations of this study}

- This study will evaluate access to an Australian nationwide bowel cancer screening programme by people with severe mental illness (SMI).

- The use of national administrative population data will ensure the study accurately identifies and includes a representative group of people with SMI and compares their treatment pathways with those of the general population.

- Screening and treatment variables will be accurately identified through the routinely collected data as will information on potential confounders/effect modifiers such as socioeconomic status, health service use and comorbidities.

- The use of antipsychotic medications as a proxy to classify those with and without SMI may misclassify some with SMI who are not taking medication as well as those with off-label antipsychotic medication use.

- The study will not have access to data on some potential effect modifiers such as smoking, alcohol or substance use.

bipolar disorder, are much greater than in the general population, ${ }^{1}$ yet only $10 \%$ of the excess is explained by suicide. ${ }^{23}$ Most of this excess mortality is due to chronic physical diseases such as cancer and cardiovascular disease. Colorectal cancer (CRC) is one of the most common causes of cancer death among those with SMI in North America, Europe and Australasia. ${ }^{2-9}$ Indeed, people with SMI are around $60 \%$ more likely to die from CRC than the general population, despite incidence rates being almost identical. ${ }^{2} 67$ The similarity in incidence rates suggests that differences in lifestyle factors, such as diet or alcohol use, are unlikely to explain most of this excess mortality. It is likely, therefore, that factors associated with barriers to 
optimal care (including screening and cancer treatment) may play a role in explaining disparities in CRC mortality in those with SMI.

Previous research in the USA indicates that members of marginalised groups, such as recent immigrants or those with lower levels of education, are less likely to report having screening procedures such as the Faecal Occult Blood Testing (FOBT) or endoscopy within the past 5 years. ${ }^{10}$ Similar disparities have been reported for CRC treatment following diagnosis. ${ }^{11}$ By contrast, there is less evidence on screening and treatment disparities for those with SMI. Data from Western Australia showed that those with any type of mental illness were less likely to have a colorectal resection or radiotherapy for CRC, as well as receive significantly fewer chemotherapy cycles than those without mental illness. ${ }^{12}$ However, there were a number of limitations to these findings. First, the study examined people with any mental illness, rather than just those with SMI. Additionally, there was a lack of information on cancer staging, which is an important determinant of treatment. The study was, therefore, unable to determine whether treatment differences were related to differences in cancer care of people with SMI as opposed to differences in cancer stage at presentation. A recent systematic review of cancer screening rates in those with mental illness showed that those with SMI are less likely to be screened for breast and cervical cancer, however, there was a lack of generalisable studies examining CRC screening rates in those with SMI. ${ }^{13}$ An Australian sample of 50-69year olds with access to universal healthcare found that those with SMI had 10\% lower rates of FOBT, after adjusting for the average number of general practitioner visits, however, they did not have data on FOBT performed as part of the National Bowel Cancer Screening Programme (NBCSP). ${ }^{14}$

The NBCSP is an Australian population-based CRC screening programme. The programme commenced in August 2006, and initially all citizens aged 50 years old were sent a kit to collect stool specimens to be returned in the mail for FOBT. They were subsequently offered rescreening every 5 years up to age 74 years. This gradually expanded to biennial screening between 2015 and 2019. ${ }^{15}$ Initial evaluations of the effectiveness of the NBCSP found that, of people diagnosed with CRC between 2006 and 2008, those who were invited to the screening programme but did not participate were over two times more likely to die of CRC than those who received screening, after adjustment for potential leadtime bias. ${ }^{16}$ However, there is a concern that these gains may widen the gap between marginalised groups and the general population as there is also evidence from Australia and overseas that people of lower socio-economic status or self-reported Indigenous status are less likely to participate in CRC screening programmes. ${ }^{17} 18$ Unfortunately, the benefit of the NBCSP for people with SMI is unknown in terms of participation, uptake of colonoscopy, stage of disease at presentation, access to cancer care and cancerrelated mortality. The Colorectal cancer Outcomes in people with Severe Mental Illness Cohort (COSMIC) Study will investigate these factors to further understand the effect of the NBCSP in this disadvantaged population. The study will also investigate treatment pathways after diagnosis to determine whether variations in the treatment that SMI patients receive, compared with those without SMI, could explain some of the disparity in CRC outcomes in this group.

The specific objectives of the COSMIC study are to:

1. Compare NBCSP participation rates between those with and without SMI (primary objective).

2. Determine whether people with SMI with a positive NBCSP screening test have a follow-up diagnostic colonoscopy at the same rate as those from the general population.

3. Calculate and compare CRC mortality rates in those with and without SMI (1) overall and (2) according to participation in the NBCSP.

4. Additionally, among New South Wales (NSW) residents diagnosed with CRC, the study aims to assess whether those with SMI: (1) are diagnosed at a later cancer stage, (2) receive surgery, or chemotherapy/ radiotherapy (or delays in their treatment) as often as those without SMI, after adjusting for cancer stage at presentation and (3) experience any differences in the above outcomes, based on participation in the NBCSP.

\section{METHODS AND ANALYSIS}

The COSMIC study will use linked routinely collected administrative national and state-based health datasets to create (1) a national cohort (objectives 1-3) and (2) a NSW cohort (objective 4). The state of NSW was selected to examine potential differences in stage at diagnosis, treatment and cancer mortality as it is the only large Australian jurisdiction that routinely collects data on estimated cancer stage at diagnosis. Linkage and analysis of data is anticipated to commence in December 2020 and the study will conclude by 30 June 2023 .

\section{Formation of the National cohort (objectives 1-3) and SMI data}

Figure 1A outlines the datasets used to form the national cohort. People with SMI will be identified using data from the Pharmaceutical Benefits Scheme (PBS). The PBS is an Australian government programme that provides access to subsidised medications to all Australian citizens and permanent residents through Medicare, Australia's universal health system. The PBS covers over $90 \%$ of all community prescription medications within Australia. ${ }^{19}$ It is likely that an even higher proportion of prescriptions for those with SMI are captured through the PBS, compared with the general population, as those with SMI are more likely to receive greater PBS subsidies. ${ }^{20}$ Second generation antipsychotics, the mainstays of treatment for SMI, require an indication-specific authority code for subsidy through the PBS. These drugs are almost solely indicated by the PBS for the treatment of either schizophrenia or 


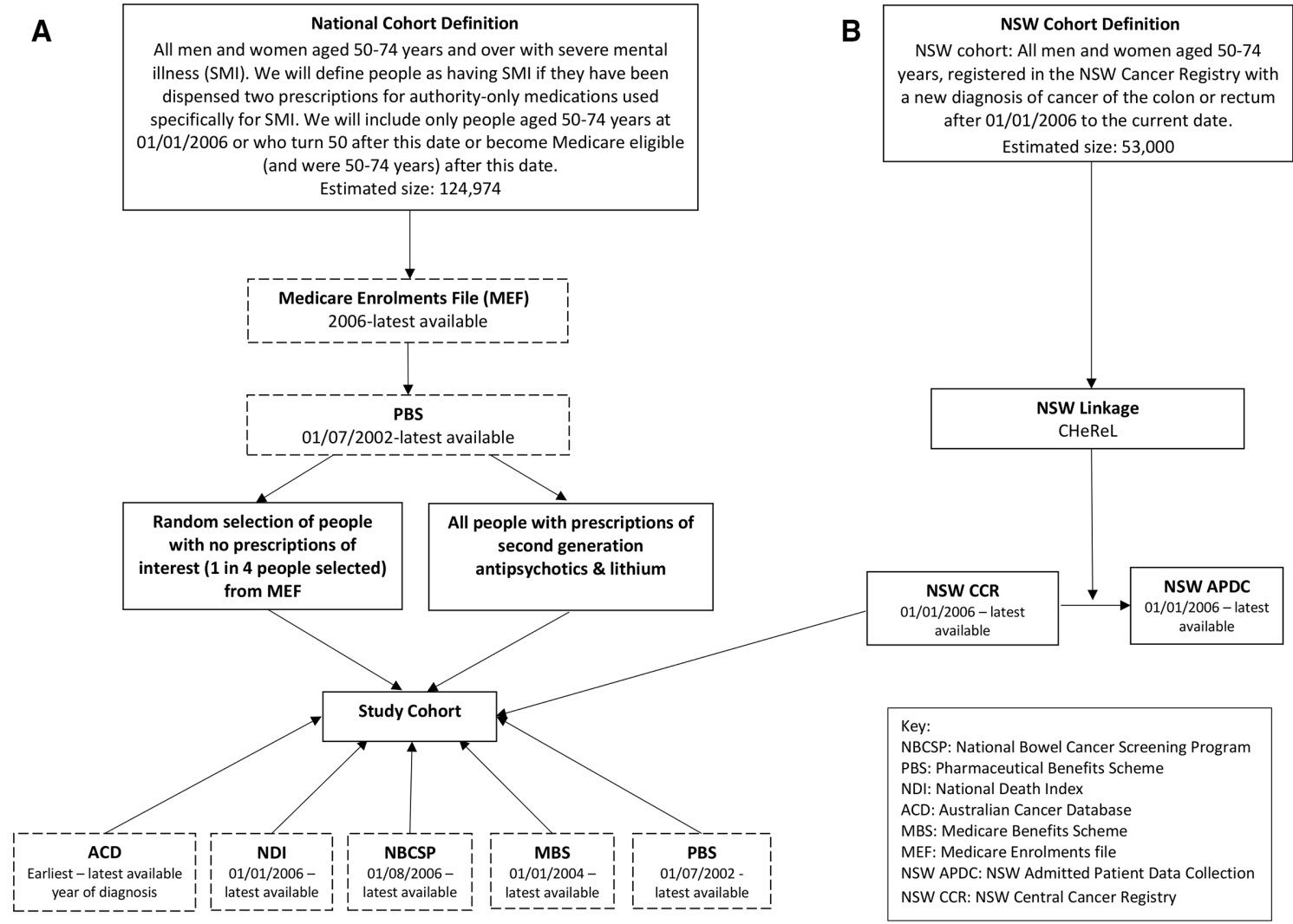

Figure 1 Flow chart of datasets used to form the (A) national cohort and (B) NSW cohort for the Colorectal cancer outcomes in people with Severe Mental IIIness cohort (COSMIC) study.

bipolar affective disorder. Lithium prescriptions will also be included, for which the specific indication is bipolar affective disorder. In this study, a person will be considered to have an SMI if they have been dispensed two or more prescriptions for authority-only medications used specifically for SMI (second generation antipsychotics and lithium; antineoplastic (ATC) codes N05AH, N05AX, N05AE, N06AX ${ }^{21}$ since 1 July 2002 (the commencement of the PBS dataset). We will include only people eligible for Medicare aged 50-74 years at 1 January 2006 or eligible people who turn 50 years after this date or people aged 50-74 years who become eligible for Medicare after this date. A population-based comparison group will be created using the Medicare Enrolments File (MEF). The MEF dataset contains a list of all people who are registered to receive Medicare benefits, including those ascertained as having SMI. From this database one in nine people will be randomly selected among those aged $50-74$ years at 1 January 2006, or eligible people who turn 50 years after this date or people aged 50-74 years who become eligible for Medicare after this date. This group will exclude people who have been dispensed a prescription for one of the specified PBS medications for SMI (as described above) between 1 July 2002 and the latest available date.

\section{Formation of the NSW cohort (objective 4)}

Figure 1B outlines the datasets used to form the NSW study cohort. The NSW cohort will include all men and women aged 50-74 years who have been registered in the NSW Central Cancer Registry with a new diagnosis of cancer of the colon or rectum (ICD-10 codes C18, C19, C20) diagnosed after 1 January 2006 to the latest available date. Identifiers from the NSW cohort will be sent for linkage with databases from the national cohort. Data from national datasets for individuals in the NSW cohort who were not included in the MEF random sample will also be requested. The PBS dataset will then be used to identify those with SMI using the same protocol as the national cohort.

\section{Outcomes and datasets}

A summary of all datasets used in this study and the key variables to be obtained from each dataset are outlined in table 1.

\section{CRC screening}

The primary outcome for this study is participation rates in the NBCSP. Data on NBCSP participation (those invited and those who returned tests) (objective 1) and screening outcomes (test results and subsequent colonoscopy uptake) (objective 2) will be obtained from the NBCSP dataset. These data have been requested for the period of 1 August 2006 (the commencement of the NBCSP) to the latest available. As NBCSP data on colonoscopy procedures can be incomplete, ${ }^{22}$ the Medicare Benefits Schedule (MBS) dataset will also be used to gain a more complete list of colonoscopy and sigmoidoscopy procedures undertaken (MBS item numbers pre- 1 November 
Table 1 Datasets used for linkage in the Colorectal cancer outcomes in people with Severe Mental IIIness (COSMIC) study

\begin{tabular}{|c|c|}
\hline Database & Description \\
\hline $\begin{array}{l}\text { Pharmaceutical Benefits } \\
\text { Scheme (PBS) }\end{array}$ & $\begin{array}{l}\text { Contains data on use of prescription medications } \\
\text { that qualify for subsidy by the Australian } \\
\text { Government. This databased will be used to identify } \\
\text { those with SMI (based on ATC codes N05AE, } \\
\text { N05AX, N05AL, N05AH and streamlined authority } \\
\text { codes specifically for schizophrenia and bipolar } \\
\text { disorder). It will also be used to examine cancer } \\
\text { treatments in the NSW cohort (ATC codes: L01, } \\
\text { L03, L04) }\end{array}$ \\
\hline Medicare Enrolments File & $\begin{array}{l}\text { Contains a list of all people within Australia (citizens } \\
\text { and permanent residents) enrolled to receive } \\
\text { Medicare benefits (Australia's universal health } \\
\text { system). This database will be used to select the } \\
\text { comparison group (those with no SMI). }\end{array}$ \\
\hline
\end{tabular}

\section{Australian Cancer Database Contains records of all new cases of malignant cancer diagnosed in Australia (exc. basal cell and squamous cell carcinomas). Cancer is a notifiable disease in all Australian states and territories.}

National Bowel Cancer Screening Programme (NBCSP)
Contains all records from the NBCSP relating to invitations, completed screening and results, follow-up colonoscopy invitations, completions and results.

\section{Key variables of interest}

PBS drug item; prescriber specialty and type; repeat prescriptions and number of scripts dispensed; quantity supplied; date of supply; streamlined authority codes

Month and year of birth; sex; postcode (for arealevel socioeconomic status)

Type of cancer (ICD-10); state of cancer registration date of diagnosis; age at diagnosis; topography; morphology; date of death; underlying cause of death

Screening programme invitation date; participation (incuding opting out of the programme) and date; screening results; colonoscopy invitation and date; colonoscopy completion; colonoscopy results (no of polyps, site and size of polyps); cancer status; primary site of cancer

$\begin{array}{ll}\text { Medicare Benefits } & \text { Contains data on Medicare-subsidised health } \\ \text { Schedule } & \text { services utilised by individuals enrolled to receive } \\ & \text { Medicare benefits (Australia's universal health } \\ \text { system). }\end{array}$

Attendance and frequency of attendance of General Practitioners and other Enhanced Primary Carers; Specialists; Clinical Psychologists and other psychologist services; sigmoidoscopy/ colonoscopy/NBCSP colonoscopy/FOBT item use

National Death Index

Contains records of all deaths registered in

Australia. Data is obtained from Registrars of Births, causes of death (ICD codes)

Deaths and Marriages in each state, the National

Coronial Information System and the Australian Bureau of Statistics

NSW Admitted Patient Data
Collection
provided by NSW public hospitals, public
psychiatric hospitals, public multipurpose services,
private hospitals and private day procedures
centres.

NSW Cancer Registry
Date of death; cause of death and contributing

Data on each episode of care including: episode of care type; facility type; date of admission and separation; principal diagnoses and additional diagnoses (to derive comorbidities); procedures undergone; source of referral and separation referrals

$\mathrm{CRC}$ diagnosis; date of diagnosis; age at diagnosis; tumour, nodes, metastases (TNM) staging and degree of spread; cancer typography and morphology; modalities of cancer care received
Records all new cases of malignant cancer (exc. basal cell and squamous cell carcinomas) and clinical data relating to episodes of cancer care for people treated for cancer in the state of NSW. This database will be used to identify all cases of CRC (ICD codes C18, C19, C20) in NSW

CRC, colorectal cancer; FOBT, Faecal Occult Blood Test; ICD-10, International Classification of Diseases V.10; NSW, New South Wales; SMI, severe mental illness; ;TNM, tumour, nodes, metastases.

2019: 32084, 32087, 32088, 32089, 32090, 32093; post1 November 2019: 32084, 32087, 32222-32229 inclusive). The MBS dataset captures data on accessed health services and procedures, and the corresponding healthcare providers that are eligible for government subsidy for those enrolled in Medicare. ${ }^{23}$ FOBT tests performed outside of the NBCSP will also be obtained through the MBS dataset (MBS item numbers: 66764, 66767, 66770).
MBS data will be obtained from 1 January 2004 to the latest available.

\section{CRC Incidence}

Cancer is a notifiable disease in all Australian states and territories. CRC incidence for the national cohort will therefore be obtained through the Australian Cancer Database. The Australian Cancer Database contains 
records of all new cases of cancer diagnosed in Australia (excluding basal cell and squamous cell carcinomas of the skin).$^{24}$ Information on the diagnoses of other cancers (for both cohorts) will also be collected to perform sensitivity analyses censoring at the time of diagnosis of other cancer, as this may influence screening practices and treatments. As such, cancer data will be obtained from the earliest available time in the dataset (1 January 1982) to the latest available date.

\section{Mortality}

CRC mortality and all-cause mortality (objective 3 ) will be ascertained from the National Death Index (NDI). The NDI database contains records of all deaths occurring in Australia since 1980, with identifying information provided regularly (approximately once a month) by all State and Territory Registrars of Births, Deaths and Marriages. ${ }^{25}$ Data on deaths from other causes will be used for censoring in analyses. Death data for both cohorts will be obtained from 1 January 2006 to the latest available.

\section{CRC stage and treatment}

For the NSW cohort, data on CRC stage (objective 4) will be obtained from the NSW Central Cancer Registry. The NSW Central Cancer Registry is the first Australian population-based cancer registry to include information on cancer stage (classified as localised, regional spread and distant spread), treatment and quality of care. ${ }^{26}$ CRC treatment (eg, resections, chemotherapy and radiation therapy) (objective 4) will be identified using the NSW Admitted Patient Data Collection (NSW APDC). The NSW APDC contains records of all admitted patient services provided by NSW public hospitals, public psychiatric hospitals, public multipurpose services, private hospitals and private day procedure centres. The PBS dataset will also be used to identify the use of ATC agents (ATC code L01). The MBS dataset will be used to identify the use of radiation therapy procedures (all MBS item numbers from category 3 therapeutic procedures; group T2 radiation oncology). All staging and treatment information will be obtained from 1 January 2006 to the latest available date.

\section{Confounders and mediators}

Frequency of medical contact may be an important mediator in the association between SMI and cancer screening. Data from the MBS will be used to capture the frequency of medical practitioner visits per year by specialty type (general practitioners, psychiatrists, oncologists, gastroenterologists, psychologists and other specialties). This data will be collected from 1 January 2004 to the latest available.

Confounders collected for consideration in the national cohort analyses include age, area-level socioeconomic status and state of residence. These variables will be obtained from the MEF dataset. Area-level socioeconomic status is classified using Socio-Economic Indexes
For Areas (SEIFA). SEIFA indices have been created by the Australian Bureau of Statistics that rank areas in Australia (based on postal area codes) according to relative socioeconomic advantage and disadvantage from 5 yearly census data of residents. ${ }^{27}$ For the NSW cohort, patient comorbidities will also be considered as a potential confounder in analyses of stage at CRC presentation and treatment received. Comorbidity data will be obtained through the NSW APDC using principal and additional diagnosis codes for hospital admissions.

\section{Data linkage}

All data for the national cohort will be extracted from all datasets and linked by the Australian Institute of Health and Welfare (AIHW) using probabilistic data linkage. For the NSW cohort, the Centre for Health Record Linkage (CHeReL) will extract and link all NSW datasets and send identifiers to the AIHW for linkage with the PBS dataset (to identify those with SMI). Once key data have been extracted and linked, all personal identifiers will be removed and each individual will be allocated a project-specific person mumber) for researchers to link deidentified individuals between datasets. The AIHW and CHeReL will load respective datasets to the Secure Unified Research Environment (SURE). SURE is a purpose-built remote-access data research laboratory, created and managed by The Sax Institute, used to enable the secure analysis of data from routinely collected registries within Australia. ${ }^{28}$

\section{Planned statistical analyses}

Time-to-event analyses will be used to calculate incidence rates and rate ratios for each outcome of interest (FOBT; colonoscopy; cancer diagnosis; surgery; chemotherapy/ radiotherapy use; and CRC and all-cause mortality), comparing those with and without SMI and the effects of the NBCSP on these outcomes. In NSW, stage at diagnosis and treatment between those with and without SMI will also be examined.

Person-years will be calculated from 2006 (the date of commencement of the NBCSP), or from the year that people become eligible for screening (ie, age 50), whichever occurs later. If an individual becomes eligible for screening before they are dispensed a medication for an SMI then the years prior to the prescription will be considered 'unexposed'.

Individuals with a previous CRC diagnosis prior to cohort entry will be excluded from all analyses as Australian guidelines recommend routine surveillance with colonoscopy rather than FOBT through the NBCSP for those with a history of CRC. ${ }^{29}$ For analyses of the primary outcome (NBCSP participation), individuals will be censored at the time of development of competing risks (incident CRC diagnosis or any cause of death), and at the end of the study period. Individuals diagnosed with other cancers during the follow-up period will be censored at the time of diagnosis in sensitivity analyses, as this may also influence screening practices and treatments. 
The models using only the national data will be adjusted for age, area-level socioeconomic status and state of residence. Adjustment by concessional status (holding a government healthcare card, or similar, to receive further subsidisation on costs of medications or health services) will also be considered as an individual-level marker of socioeconomic status. Data on the number and type of medical consultations for each person during the study period (from the MBS database) will be considered as mediators in the models. For analyses examining cancer mortality as the outcome, year of cancer diagnosis will also be adjusted for to account for improvements in CRC survival between 2006 and 2020. ${ }^{30}$ For analyses using NSW data, comorbidities will also be adjusted for by constructing a Charlson comorbidity score ${ }^{31}$ using principal and additional diagnosis codes from the NSW APDC database.

Causal mediation analyses will be conducted to examine:

1. The mediating effect of frequency of medical contact on the association between SMI and cancer screening.

2. The potential mediating effect of treatment differences or delays to treatment on the association between SMI and cancer-specific mortality.

Sensitivity analyses will be conducted to:

1. Examine whether participation in the NBCSP modifies any disparity in CRC mortality or cancer care outcomes (eg, colonoscopy rates, surgical resection rates) for people with SMI compared with all those over 50 years who participated in the programme (Australia-wide), or everyone with CRC over 50 years in NSW.

2. Explore the effect of changing the number of antipsychotic prescriptions used to define those with an SMI. Dosage information will also be collected from the PBS to exclude potential off-label use at low doses for conditions other than psychosis.

3. Examine the effect of excluding people with other mental illnesses (eg, depression) from the comparison group. This will be done using data on antidepressant use (SSRIs and SNRIs; ATC Code N06A) from the PBS dataset from 2012 onwards, when antidepressants were routinely included in the PBS.

4. Examine the effect of using hospitalisation records (available in the NSW cohort) for SMI or suicide attempts as a proxy for SMI severity (dose-response analysis) for analyses of outcomes related to cancer treatment and cancer-specific mortality.

5. Examine the effect of COVID-19 on CRC screening and treatment rates with particular focus on differences between people with SMI and the general population (if the most recently available data cover the time period including the COVID-19 pandemic).

\section{Patient and public involvement}

The COSMIC study has two patient representatives who have been involved in the project design and will have ongoing involvement throughout the study as associate investigators. One has lived experience and is a researcher in the area of physical and psychiatric comorbidity, the second has been involved, both at a state and national executive level, in the Bowelscan programme for Rotary. The Bowelscan programme is a community programme that encourages Australians most at risk to undertake FOBT for the prevention and early detection of CRC. The consumer representatives will help to communicate the findings of our research to consumer groups where appropriate and also advise on the best ways to communicate study findings to consumers. They will also provide input into how the research findings could be translated into better outcomes for people with SMI.

People with experience of SMI and CRC, and people with SMI in advocacy or peer support roles will also be consulted to provide their perspective on the lived experience and barriers and enablers to appropriate screening and cancer care services for people with SMI. These consultations will be cofacilitated by a person with lived experience of SMI to enhance engagement. The qualitative interviews will be used to further develop an understanding of the quantitative findings as well as develop ideas on how to improve access to care.

\section{DISCUSSION}

This study will be the first to examine the impact of the NBCSP on participation rates and outcomes in those with SMI. Results from this study will address both the National Advisory Council on Mental Health's call for personcentred collaborative practice to address inequities in the physical health of people with mental illness, ${ }^{32}$ as well as Cancer Australia's focus on populations who experience poor and unwarranted variations in cancer outcomes. ${ }^{33}$

This project takes advantage of Australia's data linkage infrastructure that now has the capacity to link large, national-based and state-based datasets on CRC screening, health service and medication use, cancer incidence and treatments, and mortality. As such, results will specifically identify where access and/or treatment disparities exist along the CRC cancer care pathway (from screening through to cancer care and mortality) for people with SMI in order to improve access and service use, and ultimately improve cancer survival for this disadvantaged group. If disparities exist, the results could inform efforts to ensure greater participation of people with SMI in the NBCSP. These might include alternative ways of distributing FOBT test kits, such as supplying mental health services with kits to increase opportunistic screening, and dedicated health personnel for follow-up and support.

While use of anonymised linked data from administrative databases enables access to a nationwide sample to maximise statistical power within a limited budget, there are a number of methodological considerations. The primary limitation is the inability to collect information on potential effect modifiers and confounders such as smoking, alcohol or substance use. These factors are more prevalent in those with SMI, compared with the general population, and are also associated with increased risk of CRC incidence and mortality. ${ }^{345}$ 
Adjustment for area-level data on socioeconomic and education indicators, rather than individual-level data, may also lead to residual confounding. Not adjusting for these factors may lead to an overestimation of effect sizes between SMI and CRC outcomes, particularly CRC mortality. Where possible, sensitivity analyses using quantitative bias analysis will be conducted to examine the potential effect of these covariates on effect estimates and assist with the interpretation of results.

The use of antipsychotic medications as a proxy for identifying those with SMI could lead to some misclassification bias. The essentially ubiquitous use of secondgeneration antipsychotic medications for treatment of SMI in Australia, and the requirement for specific stream-lined authority codes for prescription means that we can readily identify a large cohort of those with SMI. Therefore, using antipsychotic medications as a proxy to classify those with and without SMI is likely to have high sensitivity (ie, all those on the selected medications used to classify SMI will actually have SMI). There may, however, be some cases of SMI misclassified as having no SMI because they are not using anti-psychotic medication (lower specificity). An Australian survey of adults with psychotic illness, aged 18-64 years, who were in contact with public specialised mental health services in 20092010 found $81.6 \%$ were taking prescribed antipsychotic medications for mental health in the previous 4 weeks and had high overall compliance for antipsychotic medication (88\%). ${ }^{36}$ Medication use was also higher in older age groups compared with younger age groups. Including SMI medication prescription data from 2002 onwards, rather than just relying on current medication use, should also improve specificity. If misclassification of some cases of SMI occurs, results may be biased towards the null. However, given that SMI is rare, and the proportion not taking medication is likely to also be small, the misclassification bias is likely to have a minimal effect on results. In addition, this sample is likely to be quite representative of those with SMI in Australia, compared with studies that require individual participant consent.

There may also be an underascertainment of medications and health service use reported in some datasets, such as the PBS and MBS. The PBS database does not record data on private prescriptions (which form less than $10 \%$ of all community prescription medications ${ }^{19}$ or medication use in hospital inpatients. Similarly, the MBS does not contain information on health services, such as colonoscopies, conducted on inpatients (and some outpatients) in public hospitals. To minimise this underascertainment, where possible multiple sources of data will be used. For example, NSW cancer database, NSW APDC and PBS will be used to capture chemotherapy and immunotherapy use in NSW and the NBCSP and MBS will be used to capture colonoscopy procedures.

Although the scope of the main analyses is nationwide, most state-based cancer registries in Australia do not collect any information on cancer stage. As cancer stage is both an important outcome (are those with SMI diagnosed with CRC at a later stage than the general population?) and predictor of cancer treatment received and cancer mortality, it is an important variable to consider in analyses. To examine cancer stage, this project will use state-based cancer stage and cancer treatment data from NSW. As approximately one-third of Australia's population live in NSW, ${ }^{37}$ it is anticipated that the results from these analyses will be representative and therefore generalisable to the wider Australian population.

Finally, this study may also provide the opportunity to examine the effects of the COVID-19 pandemic, and the associated social distancing measures, on CRC screening participation rates and the timing of cancer treatment (pending available data). A study from the UK found on average a $75 \%$ reduction in urgent referrals for cancer diagnoses and $60 \%$ reduction in admissions for chemotherapy during the lockdown between March and May, 2020. ${ }^{38}$ Models examining the potential effect of this delayed diagnosis found that CRC had the highest attributable number of lives lost due to the diagnostic and treatment delays. ${ }^{39}$ It is not known if such delays have occurred in Australia, particularly among those with SMI.

\section{ETHICS AND DISSEMINATION}

The COSMIC study has been registered with the Australian New Zealand Clinical Trials Registry (ANZCTR) (ACTRN12620000781943). The study has been approved by the following ethics committees and data custodians: (1) The University of Queensland Human Research Ethics Committee (HREC) (2019000296); (2) Australian Institute of Health and Welfare Ethics Committee (E2019-5-1108); (3) Department of Health Western Australia HREC (EC00422); (4) Tasmania Health and Medical HREC (H0018672); (5) NSW Population and Health Service Research Ethics Committee (2020/ETH00414); (6) Northern Territory Department of Health (EDOC2020/79466); (7) Australian Capital Territory (2020.STE.00123). Access to the Australian Cancer Database was approved by the Queensland Cancer Alliance QLD QCCAT, South Australia Cancer Registry and Victoria Cancer Registry based on AIHW approval and protocol review.

Findings from this project will be disseminated via publications in peer-reviewed journals and presented at appropriate health service, cancer and psychiatry conferences. Given the findings are likely to also be of direct interest and relevance to health services and cancer screening, reports will also be generated for relevant stakeholders, highlighting any identified service/access gaps for people with cancer and comorbid SMI. Relevant stakeholders are likely to include the NBCSP, Cancer Australia, the National Advisory Council on Mental Health, local Divisions of General Practice and state-based cancer services and departments.

Author affiliations

${ }^{1}$ School of Public Health, The University of Queensland, Herston, Queensland, Australia 
${ }^{2}$ Population Health Department, QIMR Berghofer Medical Research Institute, Herston, Queensland, Australia

${ }^{3}$ School of Clinical Medicine, The University of Queensland, Brisbane, Queensland, Australia

${ }^{4}$ Department of Gastroenterology and Hepatology, Princess Alexandra Hospital, Woolloongabba, Queensland, Australia

${ }^{5}$ Metro South Addiction and Mental Health Service, Brisbane, Queensland, Australia ${ }^{6}$ Graduate School of Education, University of Western Australia, Perth, Western Australia, Australia

${ }^{7}$ InforMH, System Information and Analytics Branch, NSW Ministry of Health, North Ryde, New South Wales, Australia

${ }^{8}$ Northern Clinical School, Faculty of Medicine and Health, The University of Sydney, Sydney, New South Wales, Australia

${ }^{9}$ School of Allied Health, Human Services and Sport, La Trobe University, Melbourne, Victoria, Australia

${ }^{10}$ Centre for Mental Health, Melbourne School of Population and Global Health, The University of Melbourne, Melbourne, Victoria, Australia

Acknowledgements The authors wish to acknowledge Andrea McMurtrie for her support and assistance with the study. We also wish to thank our consumer representatives for their contributions to the study.

Contributors SK, SJ, DS and BJK conceived the study idea. MMP, SJ, BJK, DS, DL, GS, LB and SK provided input into the study design and research questions. MMP, SJ and SK completed the first draft of the manuscript. SJ, BJK, DS, DL, GS, LB, SK and MMP reviewed the manuscript and provided input into the final draft.

Funding This work was supported by Cancer Australia: grant number APP1157870. DS was funded in part by an NHMRC Early Career Fellowship: GNT11111136.

Competing interests None declared.

Patient and public involvement Patients and/or the public were involved in the design, or conduct, or reporting, or dissemination plans of this research. Refer to the Methods section for further details.

Patient consent for publication Not required.

Provenance and peer review Not commissioned; externally peer reviewed.

Open access This is an open access article distributed in accordance with the Creative Commons Attribution Non Commercial (CC BY-NC 4.0) license, which permits others to distribute, remix, adapt, build upon this work non-commercially, and license their derivative works on different terms, provided the original work is properly cited, appropriate credit is given, any changes made indicated, and the use is non-commercial. See: http://creativecommons.org/licenses/by-nc/4.0/.

\section{ORCID iDs}

Melinda M Protani http://orcid.org/0000-0001-6452-7571

David Lawrence http://orcid.org/0000-0003-4700-1425

Grant Sara http://orcid.org/0000-0002-3762-1711

Steve Kisely http://orcid.org/0000-0003-4021-2924

\section{REFERENCES}

1 Saha S, Chant D, McGrath J. A systematic review of mortality in schizophrenia: is the differential mortality gap worsening over time? Arch Gen Psychiatry 2007;64:1123-31.

2 Kisely S, Smith M, Lawrence D, et al. Mortality in individuals who have had psychiatric treatment: population-based study in nova Scotia. Br J Psychiatry 2005;187:552-8.

3 Lawrence D, Hancock KJ, Kisely S. The gap in life expectancy from preventable physical illness in psychiatric patients in Western Australia: retrospective analysis of population based registers. BMJ 2013;346:f2539

4 Crump C, Winkleby MA, Sundquist K, et al. Comorbidities and mortality in persons with schizophrenia: a Swedish national cohort study. Am J Psychiatry 2013;170:324-33.

5 Cunningham R, Sarfati D, Stanley J, et al. Cancer survival in the context of mental illness: a national cohort study. Gen Hosp Psychiatry 2015;37:501-6.

6 Kisely S, Forsyth S, Lawrence D. Why do psychiatric patients have higher cancer mortality rates when cancer incidence is the same or lower? Aust N Z J Psychiatry 2016;50:254-63.

7 Lawrence D, Holman CD, Jablensky AV, et al. Excess cancer mortality in Western Australian psychiatric patients due to higher case fatality rates. Acta Psychiatr Scand 2000;101:382-8.
8 Olfson M, Gerhard T, Huang C, et al. Premature mortality among adults with schizophrenia in the United States. JAMA Psychiatry 2015;72:1172-81.

9 Toender A, Munk-Olsen T, Vestergaard M, et al. Impact of severe mental illness on cancer stage at diagnosis and subsequent mortality: a population-based register study. Schizophr Res 2018;201:62-9.

10 Ward E, Jemal A, Cokkinides V, et al. Cancer disparities by race/ethnicity and socioeconomic status. CA Cancer J Clin 2004:54:78-93.

11 Shavers VL, Brown ML. Racial and ethnic disparities in the receipt of cancer treatment. J Natl Cancer Inst 2002;94:334-57.

12 Kisely S, Crowe E, Lawrence D. Cancer-Related mortality in people with mental illness. JAMA Psychiatry 2013;70:209-17.

13 Solmi M, Firth J, Miola A, et al. Disparities in cancer screening in people with mental illness across the world versus the general population: prevalence and comparative meta-analysis including 4717839 people. Lancet Psychiatry 2020;7:52-63.

14 Tuesley KM, Jordan SJ, Siskind DJ, et al. Colorectal, cervical and prostate cancer screening in Australians with severe mental illness: retrospective nation-wide cohort study. Aust N Z J Psychiatry 2019;53:550-8.

15 Australian Department of Health. National Bowel Cancer Screening Program - About the Program Australia. Australian Government Department of Health, 2019. http://www.cancerscreening.gov.au/ internet/screening/publishing.nsf/Content/about-the-program-1

16 Australian Institute of Health and Welfare. Analysis of bowel cancer outcomes for the National bowel cancer screening program. Canberra: AlHW, 2014

17 Christou A, Katzenellenbogen JM, Thompson SC. Australia's national bowel cancer screening program: does it work for Indigenous Australians? BMC Public Health 2010;10:373.

18 von Wagner $\mathrm{C}$, Baio $\mathrm{G}$, Raine $\mathrm{R}$, et al. Inequalities in participation in an organized national colorectal cancer screening programme: results from the first 2.6 million invitations in England. Int $J$ Epidemiol 2011;40:712-8

19 Australian Department of Health. Australian statistics on medicines 2011. Canberra, Australia: Australian Department of Health, 2013.

20 Sweeney S, Air T, Zannettino L, et al. Psychosis, socioeconomic disadvantage, and health service use in South Australia: findings from the second Australian national survey of psychosis. Front Public Health 2015;3:259.

21 WHO Collaborating Centre for Drug Statistics Methodology. Guidelines for ATC classification and DDD assignment 2020. Oslo, Norway, 2019.

22 Australian Institute of Health and Welfare. National bowel cancer screening program: monitoring report 2019. cancer series no 125 cat no can 125. Canberra: AlHW, 2019.

23 Australian Department of Health. Medicare benefits schedule (MBS). Canberra, Australia: Commonwealth of Australia, 2020. http://www. mbsonline.gov.au/internet/mbsonline/publishing.nsf/Content/Home

24 Australian Institute of Health and Welfare. Australian cancer database (ACD) Australia: AlHW, 2019. Available: https://www.aihw.gov.au/ about-our-data/our-data-collections/australian-cancer-database [Accessed 2 Jul 2020].

25 Australian Institute of Health and Welfare. About national death index Australia: AlHW, 2020. Available: https://www.aihw.gov.au/aboutour-data/our-data-collections/national-death-index/about-nationaldeath-index [Accessed 2 Jul 2020].

26 Cancer Institute NSW. Nsw cancer registry NSW. Australia: Cancer Institute NSW, 2020. https://www.cancer.nsw.gov.au/data-research/ cancer-related-data/request-unlinked-unit-record-data-for-research/ nsw-cancer-registry

27 Australian Bureau of Statistics. Socio-Economic indexes for areas Canberra. Australia: ABS, 2018. https://www.abs.gov.au/websitedbs/ censushome.nsf/home/seifa

28 The Sax Institute. Sure Australia: the sax Institute (acn 095542886 (Institute), 2005. Available: https://www.saxinstitute.org.au/our-work/ sure/ [Accessed 2 Jun 2020].

29 Australian Department of Health. Managing bowel screening for participants with special conditions Australia: Commonwealth of Australia, 2020. Available: https://www.health.gov.au/initiatives-andprograms/national-bowel-cancer-screening-program/managingbowel-screening-for-participants/managing-bowel-screening-forparticipants-with-special-conditions [Accessed 19 Feb 2021].

30 Australian Institute of Health and Welfare. Cancer data in Australia. Canberra: AlHW, 2020. https://www.aihw.gov.au/reports/cancer/ cancer-data-in-australia. (accessed 19 Feb 2021).

31 Charlson ME, Pompei P, Ales KL, et al. A new method of classifying prognostic comorbidity in longitudinal studies: development and validation. J Chronic Dis 1987;40:373-83. 
32 National Advisory Council of Mental Health. A mentally healthy fuure for all Australians, a discussion paper. Canberra, Australia: NACMH, 2009.

33 Cancer Australia. Cancer Australia strategic plan 2014-2019. Surry Hills, NSW: Cancer Australia, 2014

34 Cai S, Li Y, Ding Y, et al. Alcohol drinking and the risk of colorectal cancer death: a meta-analysis. Eur J Cancer Prev 2014:23:532-9.

35 Walter V, Jansen L, Hoffmeister M, et al. Smoking and survival of colorectal cancer patients: systematic review and meta-analysis. Ann Oncol 2014;25:1517-25.

36 Morgan V, Waterreus A, Jablensky A. People living with psychotic illness 2010 - Report on the second Australian national survey. Canberra: Australian Government, 2011.
37 Australian Bureau of Statistics. 3101.0 - Australian Demographic Statistics, Dec 2019 Canberra. Australia: ABS, 2020. https:// www.abs.gov.au/AUSSTATS/abs@.nsf/Lookup/3101.0Main+ Features1Dec\%202019?OpenDocument

38 Lai AG, Pasea L, Banerjee A. Estimating excess mortality in people with cancer and multimorbidity in the COVID-19 emergency.. medRxiv 2020.

39 Sud A, Torr B, Jones ME, et al. Effect of delays in the 2-week-wait cancer referral pathway during the COVID-19 pandemic on cancer survival in the UK: a modelling study. Lancet Oncol 2020;21:1035-44. 